\title{
Etats de mer et circulation océanique en zone côtière
}

\author{
Fabrice Ardhuin et Nicolas Rascle \\ SHOM, Centre Militaire d'Océanographie, \\ 13 rue du Chatellier, 29609 Brest
}

\begin{abstract}
Résumé
La moyenne Lagrangienne généralisée des équations du mouvement permet une partition de la quantité de mouvement (QDM) en une composante associée aux ondes courtes, le reste étant associé à la circulation et ondes longues. Une approximation au second ordre en cambrure des vagues donne un jeu d'équation pour l'évolution de cette deuxième partie. Le forçage par les vagues fait intervenir directement un terme de pression moyenne induite par les vagues, et un forçage par la dissipation des vagues. Ce jeu d'équations est intégré sur la verticale et comparé avec d'autres jeux d'équations existants. Les effets des processus paramétrés par des termes source dans l'équation d'évolution de l'action sont décrits. Des premières applications sont données pour des cas bidimensionnels par un calcul numérique utilisant une version modifiée du modèle de circulation ROMS.
\end{abstract}

\begin{abstract}
Sea state and coastal ocean circulation Based on a generalized Lagrangian mean of the equations of motion, a second order approximation in powers of the wave slope yields an explicit set of evolution equations for the current momentum. Wave forcing in that that system takes the form of the mean wave-induced pressure, and a body force arising from the dissipation of waves. This set of equations is integrated over the vertical and compared to other existing sets of equations. The effects of each process that is parameterized by source term in the wave action balance are described. First applications are given for two-dimensional cases, using a modified version of the ocean circulation model ROMS.
\end{abstract}

\section{Mots clés :}

tensions de radiation, moyenne Lagrangienne généralisée, courants 3D

\section{Introduction}

De nombreux travaux récents ${ }^{23,17,16}$ visent à établir des équations tridimensionnelles pour l'écoulement moyen forcé par l'état de la mer. En effet la circulation littorale, au voisinage de la zone de déferlement, est en grande partie forcée par les vagues ${ }^{6}$. Sur le plateau continental interne il est aussi probable que les vagues aient un effet important ${ }^{1} 4$. Au large, les vagues sont le principal intermédiaire de la dérive induite par le vent ${ }^{1} 2$ et du mélange à la surface de l'océan ${ }^{1}$. Il est donc indispensable de donner une description cohérente des interactions entre circulation océanique et état de mer pour toute étude sérieuse de la dynamique océanique 
superficielle ou côtière. Par ailleurs cette description, pour ce qui est des grandes échelles, fait nécessairement appel à des moyennes sur des échelles de temps et d'espace plus grandes que la période et la longueur d'onde des vagues.

Il faut donc régler deux problèmes essentiels. Le premier est la mobilité de l'interface air-mer qui conduit à un mélange d'air et d'eau entre les creux et crêtes si une moyenne Eulérienne (au point fixe) est choisie. Le second est la nature très différentes des mouvements rapides qui combinent une dynamique ondulatoire, qui se propagent très vite, et turbulente, qui diffusent plus lentement. Le premier problème conduit à utiliser des moyennes qui suivent au moins l'interface air-mer, tandis que le second conduit à séparer la dynamique des vagues de la dynamique de la circulation. Ce double choix est rationnel, il est cependant différent de la plupart des approches suivies jusqu'à présent. En effet le choix fait par McWilliams et coll. ${ }^{16}$ remplace la moyenne suivant l'interface par une moyenne Eulérienne (tout en gardant la séparation vagues-courant). Près de la surface, l'approche de ces auteurs n'a un sens que de manière asymptotique, pour une amplitude des vagues tendant vers zéro.

Enfin, le choix inverse a été fait par Walstra et coll. ${ }^{23}$ et Mellor ${ }^{17}$. Ce dernier choix mélange la quantité de mouvement des vagues et de la circulation moyenne au sens ou le vecteur vitesse horizontale $\mathrm{U}$ que ces auteurs proposent de calculer est en fait la vitesse de dérive qui comprend la dérive de Stokes $\mathbf{U}_{\mathbf{s}}$. Or cette dernière n'est pas mélangée par la turbulence, et il faut donc prendre garde de soustraire $\mathbf{U}_{\mathbf{s}}$ de $\mathbf{U}$ avant de calculer la clôture turbulente, par exemple. Il parait plus simple de calculer directement l'évolution de $\widehat{\mathbf{u}}=\mathbf{U}-\mathbf{U}_{\mathbf{s}}$. Nous reprenons ici la théorie exposée par Ardhuin ${ }^{3}$ en discutant plus spécifiquement des effets de processus contribuant à l'évolution des vagues, en particulier pour les équations intégrées sur la verticale.

\section{Théorie}

\subsection{Moyenne Lagrangienne généralisée (GLM)}

Une équation d'évolution 'GLM' pour $\widehat{\mathbf{u}}$ a été donnée par Andrews et McIntyre², avec pour seule hypothèse une moyenne nulle pour les oscillations du vecteur déplacement induit par les vagues $\xi=\left(\xi_{1}, \xi_{2}, \xi_{3}\right)$, ce qui peut toujours être imposé, et une inversibilité de la transformation $\mathbf{x} \rightarrow \Xi(\mathbf{x})=\mathbf{x}+\xi$. Ces équations sont donc extrêmement générales (non-linéarité, vorticité ...), mais implicites, au sens ou le mouvement induit par les vagues doit être déterminé par ailleurs, par exemple par McWilliams et coll. ${ }^{16}$.

Pour tout opérateur de moyenne Eulérienne, on note $\overline{\phi(\mathbf{x}, t)}$ la moyenne de $\phi(\mathbf{x}, t)$ (ce peut être une moyenne sur des phases, des réalisations, une moyenne temporelle ou spatiale). La définition de la moyenne GLM de $\phi$ est $\overline{\phi(x, t)}^{L}=$ $\overline{\phi(\mathbf{x}+\xi, t)}$. Ainsi, la moyenne GLM est différente de la moyenne Eulérienne. La différence entre ces deux moyennes est la correction de Stokes ${ }^{2}$. Sous les creux des vagues, la correction de Stokes de la vitesse est la dérive de Stokes

$$
\overline{\mathbf{u}}^{L}-\bar{u}=\mathbf{U}_{s} .
$$




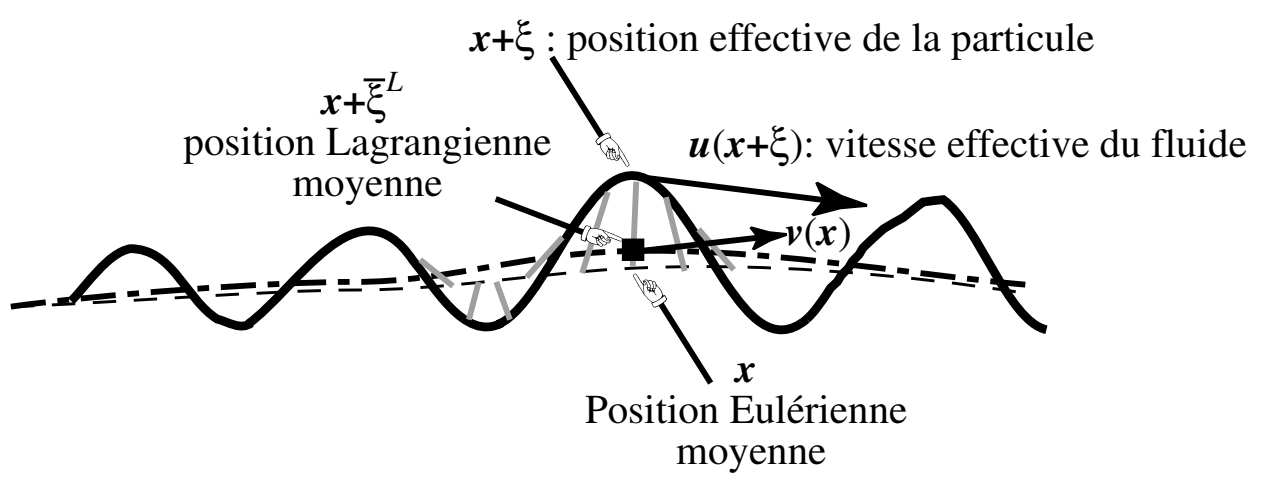

fond

FIG. 1 - Schéma de principe de la moyenne Lagrangienne généralisée.

La moyenne GLM au point $\mathrm{x}$ est en fait une moyenne sur les valeurs du champ en $\mathbf{x}+\xi$. La position de la surface est figurée par le trait plein épais et les déplacements $\xi$ de la surface sont symbolisés par les barres grises.

$\overline{\mathbf{u}}^{L}$ s'interprète simplement comme la vitesse de dérive moyenne des particules d'eau. De manière plus générale, pour un champ continuement différentiable $\phi$, la correction de Stokes est donnée par ${ }^{9}$

$$
\bar{\phi}^{L}=\bar{\phi}+\overline{\xi_{j} \frac{\partial \phi}{\partial x_{j}}}+\frac{1}{2} \overline{\xi_{j} \xi_{k}} \frac{\partial^{2} \bar{\phi}}{\partial \xi_{j} \partial \xi_{k}}+O\left(\varepsilon_{1}^{3}\right),
$$

avec $\varepsilon_{1}$ représentant la cambrure des vagues ou l'amplitude relative $a / h$, supposées du même ordre.

En définissant $\mathbf{X}$ comme la divergence du tenseur de Reynolds (flux de quantité de mouvement lié à la turbulence), on peut appliquer la moyenne Lagrangienne généralisée (GLM) aux équations de Navier-Stokes en moyenne de Reynolds (RANS). Afin de simplifier la discussion, nous utiliserons ici la forme des équations GLM donnée par Groeneweg ${ }^{8}$ pour un densité de l'eau $\rho_{w}$ constante, ce qui supprime, entre autres, les termes liés à la thermodynamique du fluide. La conservation de la composante $i=1,2,3$ de la quantité de mouvement moyenne $\widehat{u}_{i}=\bar{u}_{i}^{L}-P_{i}$ est donnée par

$$
D^{L}\left(\bar{u}_{i}^{L}-P_{i}\right)+\epsilon_{i 3 j} f_{3} \bar{u}_{j}^{L}+\frac{1}{\rho_{w}} \frac{\partial \bar{p}^{L}}{\partial x_{i}}-\bar{X}_{i}^{L}+g \delta_{i 3}=\frac{\partial}{\partial x_{i}}\left[\frac{1}{2} \overline{u_{j}^{l} u_{j}^{l}}\right]+P_{j} \frac{\partial \bar{u}_{j}^{L}}{\partial x_{i}},
$$

où la dérivée Langragienne $D^{L}=\left(\partial / \partial t+\overline{\mathbf{u}}^{L} \cdot \nabla\right)$ est une dérivée en suivant le fluide à la vitesse Langragienne moyenne $\bar{u}^{L}$, et $u_{j}^{l}=u_{j}^{\xi}-\bar{u}_{j}^{L}$ est la perturbation Lagrangienne de la composante $j$ du vecteur vitesse. La gravité $g$ n'intervient que pour la composante verticale $i=3$ et seule la composante verticale du paramètre de Coriolis $f_{3}$ a été retenue. Le symbole $\epsilon_{i_{1} i_{2} i_{3}}$ représente la signature de permutation des indices $i_{1}, i_{2}$ et $i_{3}$ et est utilisé ici pour exprimer le produit vectoriel entre le 
vecteur rotation de la terre et le courant de dérive. Par ailleurs, $P_{i}$ est la composante suivant $i$ de la pseudo quantité de mouvement des vagues ${ }^{9}$. De manière exacte aux ordres $\varepsilon_{1}^{2}$ et $\varepsilon_{2}^{0}, P_{i}=U_{s i}$ est la dérive de Stokes, qui est horizontale $\left(U_{s 3}=0\right)$, avec $\varepsilon_{2}$ est un petit paramètre représentant la non-homogénéité du champ de vagues et du milieu (pente du fond, variation horizontale et verticale du courant, gradients de l'amplitude des vagues ...). $U_{s i}$ est la vitesse moyenne de dérive des particules fluides induites par le mouvement rapide des vagues.

\subsection{Approximation : équations $g \operatorname{lm} 2$-RANS}

Les vagues forcent l'évolution de la circulation (3) par le terme de pression $\bar{p}^{L}$, le terme de Bernouilli $\overline{u_{j}^{l} u_{j}^{l}}$, et enfin le terme $P_{j} \partial \bar{u}_{j}^{L} / \partial x_{i}$. Par ailleurs, la force de Coriolis s'applique au courant de dérive qui englobe la dérive de Stokes. Ce dernier forçage 'Stokes-Coriolis' a tendance à générer un courant Eulérien dont le transport moyen annule, en moyenne, celui de la dérive de Stokes ${ }^{9,24,19}$.

En pratique on peut estimer ces termes par une approximation du mouvement des vagues. Au second ordre en cambrure on obtient des équations baptisées "glm2RANS" avec un forçage explicite par le spectre de l'état de la mer. L'avantage de ces équations est que les termes quadratiques dans (3) ne sont pas affectés par les corrections de premier ordre en $\varepsilon_{2}$ (pente du fond, gradient d'amplitude des vagues), car ces corrections sont en quadrature avec les termes d'ordre $\varepsilon_{2}^{0}$. Il s'agit là d'un grande simplification par rapport aux équations de Mellor ${ }^{1} 7$. Par contre, le cisaillement vertical du courant introduit des termes correctifs ${ }^{1} 6$ qui sont en phase avec les termes d'ordre $\varepsilon_{2}^{0}$. Le cisaillement vertical induit donc une modification ${ }^{17,3}$ de $\overline{u_{j}^{l} u_{j}^{l}}$ et $\mathbf{P}$ que nous négligerons ici. On utilise donc les vitesses et pressions $\widetilde{u}_{\alpha}, \widetilde{w}, \widetilde{p}$, issues de la théorie d'Airy pour des vagues uniformes sur fond plat.

Nous allons calculer les différents termes induits par les vagues, d'abord pour une onde monochromatique d'amplitude $a$ dont la variance de l'élévation est $E=a^{2} / 2$. On fera ensuite la superposition des composantes. Considérons d'abord le terme de pression. On définit les notations suivantes, $F_{C S}=\cosh [k(z+h)] / \sinh (k D)$, $F_{S S}=\cosh [k(z+h)] / \sinh (k D), F_{S C}=\sinh [k(z+h)] / \cosh (k D)$, avec $-h$ la côte moyenne du fond. La profondeur moyenne est $D=h+\bar{\zeta}$, avec $\zeta$ la côte de la surface libre.

En utilisant la relation de dispersion $\sigma^{2}=g k \tanh (k D)$ et le fait que $\overline{\widetilde{u}_{\alpha} \widetilde{u}_{\alpha}-\widetilde{w}^{2}}=\sigma^{2}\left(F_{C S} F_{C S}-F_{S S} F_{S S}\right)$ est indépendant de $z$ à l'ordre $\varepsilon_{1}^{2}$, on peut ajouter $0.5 \partial\left(\widetilde{u}_{\alpha} \widetilde{u}_{\alpha}-\widetilde{w} \widetilde{w}\right) / \partial z$ à l'équation sur la verticale (3) pour obtenir, toujours à l'ordre $\varepsilon_{1}^{2}$,

$$
\frac{\partial \widehat{w}}{\partial t}+\widehat{w} \frac{\partial \widehat{w}}{\partial z}+\left(\widehat{u}_{\alpha}+U_{s \alpha}\right) \frac{\partial \widehat{w}}{\partial x_{\alpha}}+\frac{1}{\rho_{w}} \frac{\partial \bar{p}^{L}}{\partial z}+g=\frac{\partial{\widetilde{u_{\alpha}}}_{\widetilde{u}_{\alpha}}}{\partial z}+U_{s \beta} \frac{\partial \widehat{u}_{\beta}}{\partial z},
$$

qui se transforme en

$$
\frac{\partial}{\partial z}\left(\frac{\bar{p}^{L}}{\rho_{w}}+g z-\sigma^{2} E F_{C S} F_{C S}\right)=-\frac{\partial \widehat{w}}{\partial t}-\widehat{w} \frac{\partial \widehat{w}}{\partial z}-\left(\widehat{u}_{\alpha}+U_{s \alpha}\right) \frac{\partial \widehat{w}}{\partial x_{\alpha}}+U_{s \beta} \frac{\partial \widehat{u}_{\beta}}{\partial z} .
$$


On peut négliger le membre de droite ${ }^{8}$ pour obtenir, à partir de la condition de continuité de la pression en surface $\overline{p(\zeta)}^{L}=\bar{p}_{a}$,

$$
\bar{p}^{L}=\bar{p}^{H}+\rho_{w} g k E F_{C C} F_{C S},
$$

avec $\bar{p}^{H}$ la pression hydrostatique définie égale à la pression atmosphérique moyenne en surface, $\bar{p}^{H}=\rho_{w} g(\bar{\zeta}-z)+\bar{p}_{a}$. Ardhuin ${ }^{3}$ a montré que ce terme de pression est identique à celui donné par McWilliams et coll. ${ }^{1} 6$, même lorsque le membre de droite de (5) est pris en compte.

En notant la vitesse quasi-Eulérienne $\widehat{\mathbf{u}}=\overline{\mathbf{U}}^{L}-\mathbf{P}$, et en considérant maintenant des vagues aléatoires de spectre $E(\mathbf{k})$, l'équation (3) devient, pour la vitesse sur la direction horizontale $\alpha=1,2$,

$$
\begin{aligned}
\frac{\partial \widehat{u}_{\alpha}}{\partial t} & +\left(\widehat{u}_{\beta}+\widehat{U}_{s \beta}\right) \frac{\partial \widehat{u}_{\alpha}}{\partial x_{\beta}}+\widehat{w} \frac{\partial \widehat{u}_{\alpha}}{\partial z}+\epsilon_{\alpha 3 \beta} f_{3} \bar{u}_{\alpha}^{L}+\frac{1}{\rho_{w}} \frac{\partial \bar{p}^{H}}{\partial x_{\alpha}} \\
& =-\frac{\partial S^{p}}{\partial x_{\alpha}}+U_{s \beta} \frac{\partial \widehat{u}_{\beta}}{\partial x_{\alpha}}+\bar{X}_{\alpha}^{L}+O\left(\varepsilon_{1}^{2} \varepsilon_{2}\right),
\end{aligned}
$$

avec

$$
S^{p}=g \int_{\mathbf{k}} k \frac{E(\mathbf{k})}{\sinh 2 k D} \mathrm{~d} \mathbf{k} .
$$

Ce terme $S^{p}$ correspond à la pression Eulérienne moyenne ${ }^{1} 6$ induite par les vagues et il est uniforme sur la verticale.

Un paramétrage possible de la source de quantité de mouvement provenant des mouvements diabatiques est

$$
\bar{X}_{\alpha}^{L}=\frac{\partial R_{\alpha \beta}}{\partial x_{\beta}}+\frac{\partial}{\partial z}\left(K_{z} \frac{\partial \widehat{u}_{\alpha}}{\partial z}\right)-T_{\alpha}^{\mathrm{dis}},
$$

avec $R_{\alpha \beta}$ le tenseur de Reynolds turbulent horizontal. $T_{\alpha}^{\text {dis }}$ est flux de quantité de mouvement des vagues vers la circulation moyenne, liés à la dissipation des vagues par le déferlement et l'interaction entre vagues et turbulence ${ }^{4}$.

Les termes d'advection peuvent être recombinés ${ }^{8}$ pour faire apparaître une force de vortex ${ }^{7,21}$, qui est le produit vectoriel de la dérive de Stokes par la composante verticale de la vorticité $\omega_{3}$,

$\frac{\partial \widehat{u}_{\alpha}}{\partial t}+\widehat{u}_{\beta} \frac{\partial \widehat{u}_{\alpha}}{\partial x_{\beta}}+\widehat{w} \frac{\partial \widehat{u}_{\alpha}}{\partial z}+\epsilon_{\alpha 3 \beta}\left[f_{3} \widehat{u}_{\alpha}+\left(f_{3}+\omega_{3}\right) U_{s \beta}\right]+\frac{1}{\rho_{w}} \frac{\partial \bar{p}^{H}}{\partial x_{\alpha}}=-\frac{\partial}{\partial x_{\alpha}} \frac{S^{J}}{\rho_{w} D}+\bar{X}_{\alpha}^{L}$.

Par ailleurs l'équation de conservation de la masse s'écrit ${ }^{9}$,

$$
\frac{\partial\left(\rho_{w} J\right)}{\partial t}+\frac{\partial\left(\rho_{w} J \bar{u}_{\alpha}^{L}\right)}{\partial x_{\alpha}}+\frac{\partial\left(\rho_{w} J \bar{w}^{L}\right)}{\partial z}=0,
$$

ou $J$ est le Jacobien de la transformation des coordonnées cartésiennes en positions Lagrangiennes moyennes. Le champ de vitesse $\bar{u}^{L}$ est donc généralement divergent 
mais peut se transformer en champ non-divergent en corrigeant de l'effet de dilatation verticale du GLM.

En effet, la moyenne GLM déplace légèrement les coordonnées sur la verticale, en particulier la surface libre GLM est légèrement au-dessus de sa moyenne Eulérienne. En utilisant (2), on obtient $\bar{\zeta}^{L}=\bar{\zeta}+k E / \tanh (k D)$, pour une onde monochromatique. Une utilisation précise de (10) demande donc une transformation de coordonnées ${ }^{3}$ par exemple pour avoir une coordonnée verticale $z$ classique, variant de $-h$ au fond à $\bar{\zeta}$ en surface. L'équation (7) est mathématiquement identique à celle obtenue par moyenne Eulérienne par McWilliams et coll. ${ }^{1} 6$ en rajoutant les termes diabatiques. Cela permet d'interpréter cette dernière jusqu'à la région entre creux et crêtes où la moyenne Eulérienne est indéfinie, il reste à vérifier si cette dilatation verticale de la moyenne GLM, qui n'existe pas dans la moyenne Eulérienne de McWilliams et coll. ${ }^{1} 6$, a un effet ou non sur la dynamique.

\subsection{Equations intégrées sur la verticale}

Afin de mieux comprendre les particularités des équations du mouvement déterminées ci-dessus, et les replacer dans le contexte des travaux antérieurs, il est instructif de déterminer l'équation correspondant à la quantité de mouvement intégrée sur la verticale, obtenue en multipliant (10) par le Jacobien $J$ de la moyenne GLM avant d'intégrer de $z=-h$ à $z=\bar{\zeta}^{L}$. Pour les termes qui sont déjà d'ordre $\varepsilon_{1}^{2}$, comme la force de vortex, cela revient, à l'ordre $\varepsilon_{1}^{2}$, à intégrer simplement (7) de $-h$ à $z=\bar{\zeta}$. Enfin, pour les termes uniformes sur la verticale (e.g. $\left.\partial p^{H} / \partial x_{\alpha}=\partial \bar{\zeta} / \partial x_{\alpha}\right)$, l'introduction du Jacobien compense exactement la continuation de l'intégrale entre $\bar{\zeta}$ et $\bar{\zeta}^{L}$. Ainsi

$$
\begin{aligned}
-\int_{-h}^{\bar{\zeta}^{L}} J \frac{\partial S^{p}}{\partial x_{\alpha}} \mathrm{d} z & =-\int_{-h}^{\bar{\zeta}} \frac{\partial S^{p}}{\partial x_{\alpha}} \mathrm{d} z=-\frac{\partial}{\partial x_{\alpha}}\left(D S^{p}\right)+S^{p}\left(\frac{\partial \bar{\zeta}}{\partial x_{\alpha}}+\frac{\partial h}{\partial x_{\alpha}}\right) \\
& =-\frac{\partial}{\partial x_{\alpha}}\left(D S^{p}\right)+S^{p} \frac{\partial D}{\partial x_{\alpha}}=-D \frac{\partial S^{p}}{\partial x_{\alpha}}
\end{aligned}
$$

ce qui est exactement le terme de pression en deux dimensions (2D) donné par $\mathrm{Smith}^{21}$. L'intégration verticale des équations 3D donne donc

$$
\begin{aligned}
\frac{\partial M_{\alpha}^{m}}{\partial t}+ & \int_{-h}^{\bar{\zeta}^{L}} \rho_{w} \widehat{u}_{\alpha} \widehat{u}_{\beta} \mathrm{d} z+\epsilon_{\alpha 3 \beta} f_{3} M_{\beta}^{m}+D \frac{\partial}{\partial x_{\alpha}}\left(\rho_{w} g \bar{\zeta}+p_{a}\right) \\
= & -\epsilon_{\alpha 3 \beta}\left(f_{3}+\Omega_{3}\right) M_{\beta}^{w}-u_{A \alpha} \frac{\partial M_{\beta}^{w}}{\partial x_{\beta}}-\left(U_{s \beta} J \widehat{u}_{\alpha}\right)_{z=\bar{\zeta}^{L}} \frac{\partial \bar{\zeta}^{L}}{\partial x_{\beta}}-D \rho_{w} \frac{\partial S^{p}}{\partial x_{\alpha}} \\
& +X_{\alpha}^{\mathrm{int}}
\end{aligned}
$$

avec $M_{\alpha}^{m}$ la composante $\alpha$ du transport de masse induit par le courant, intégré sur la verticale $M_{\alpha}^{m}=\int_{-H}^{\bar{\zeta}^{L}} \rho_{w} J \widehat{u}_{\alpha} \mathrm{d} z$. La source de quantité de mouvement $X^{\text {int }}$ est simplement le flux net de quantité de mouvement (q.d.m.) moyenne (hors q.d.m. des vagues) en surface et au fond, à laquelle s'ajoute la source de q.d.m. dans la 
colonne d'eau du fait des interaction diabatiques entre les vagues et la circulation (source de q.d.m. du fait de la dissipation des vagues),

$$
X_{\alpha}^{\mathrm{int}}=\tau_{a \alpha}-\tau_{\alpha}^{\mathrm{in}}-\tau_{\alpha}^{\mathrm{dis}}-\tau_{b \alpha},
$$

où $\tau_{a \alpha}$ et $\tau_{b \alpha}$ sont les composantes dans la direction $\alpha$ des flux nets de q.d.m. de l'atmosphere vers l'ocean, et de l'ocean vers le fond (généralement appelés 'tension de vent' et 'tension de fond'). $\tau_{\alpha}^{\text {in }}$ est la composante correspondante de la fraction de la tension de vent qui contribue à la croissance des vagues, tandis que $\tau_{\alpha}^{\text {dis }}=$ $\int_{-h}^{\bar{\zeta}} T_{\alpha}^{\mathrm{dis}} \mathrm{d} z$ est la source de q.d.m. correspondant à la dissipation des vagues.

L'équation (13) est cohérente avec les équations 2D connues, à la différence que le cisaillement vertical du courant est maintenant explicitement pris en compte. En particulier les vitesse de courant de surface utilisées par Smith ${ }^{21}$ sont maintenant remplacées par la vitesse d'advection ${ }^{13,19}, u_{A \alpha}=\int_{-h}^{\bar{\zeta}^{L}} J \widehat{u}_{\alpha} k \cosh [2 k(z+$ $h)] / \sinh (2 k D) \mathrm{d} z$, dont la vorticité est $\Omega_{3}$. Le terme adiabatique $S^{J} / D$ dans l'équation du mouvement moyen (10) est uniforme sur la verticale. L'équation (13) diffère aussi de l'équation (2.28) de $\operatorname{Smith}^{21}$ par le terme $\left(U_{s \beta} J \widehat{u}_{\alpha}\right)_{z=\bar{\zeta}^{L}}$, qui est toutefois généralement faible.

\section{Applications}

Une première application de (7) a consisté a vérifier les équilibres connus de décôte et surcôte pour une houle normalement incidente sur une plage (figure 2). Les équations du mouvement sont résolues avec une version adaptée 2D-crosshore du modèle de circulation océanique ROMS ${ }^{18}$. Le domaine choisi couvre un plateau continental en pente uniforme de $400 \mathrm{~m}$ de fond à $400 \mathrm{~km}$ de la côte. La houle est monochromatique avec une énergie correspondant à une hauteur significative $H s=3 \mathrm{~m}$, et une période $T=12 \mathrm{~s}$. La dissipation de cette houle sous l'effet du déferlement est calculée avec le modèle de Thornton et $\mathrm{Guza}^{22}$ dont les paramètres libres sont $B=1$ et $\gamma$ calculé en fonction de la pente des vagues ${ }^{5}$.

Par ailleurs, nous avons aussi étudié la transition entre la dynamique 'océanique', étudié par Xu et Bowen ${ }^{24}$ avec une dérive de Stokes compensée par un courant via la force de Coriolis, et la dynamique littorale où le courant de retour est accéléré par la surcôte induite par les vagues. Cette transition est illustrée par la figure 3 . Ces premiers calculs ont été réalisés avec une viscosité turbulente $K_{z}=0.01 \mathrm{~m} \mathrm{~s}^{-1}$ constante, ce qui permet de mieux comparer ces résultats à ceux de Xu et Bowen ${ }^{24}$. La prise en compte d'un spectre réaliste de l'état de mer et d'un paramétrage réaliste de la turbulence a été faite par ailleurs pour le cas homogène horizontalement ${ }^{19}$.

Sur la figure 3, la différence entre les profils de courant avec force de Coriolis (traits pleins) et sans force de Coriolis (pointillés) montre bien la transition entre les deux phénomènes responsables du courant de retour que sont le forçage StokesCoriolis au large, et la surcôte induite par les tensions de radiation sur la plage. Les profils de courants sont encore indicatifs et demandent à être améliorés par une représentation réaliste de la turbulence, ainsi qu'une prise en compte des effets non-linéaires des vagues déferlantes. 

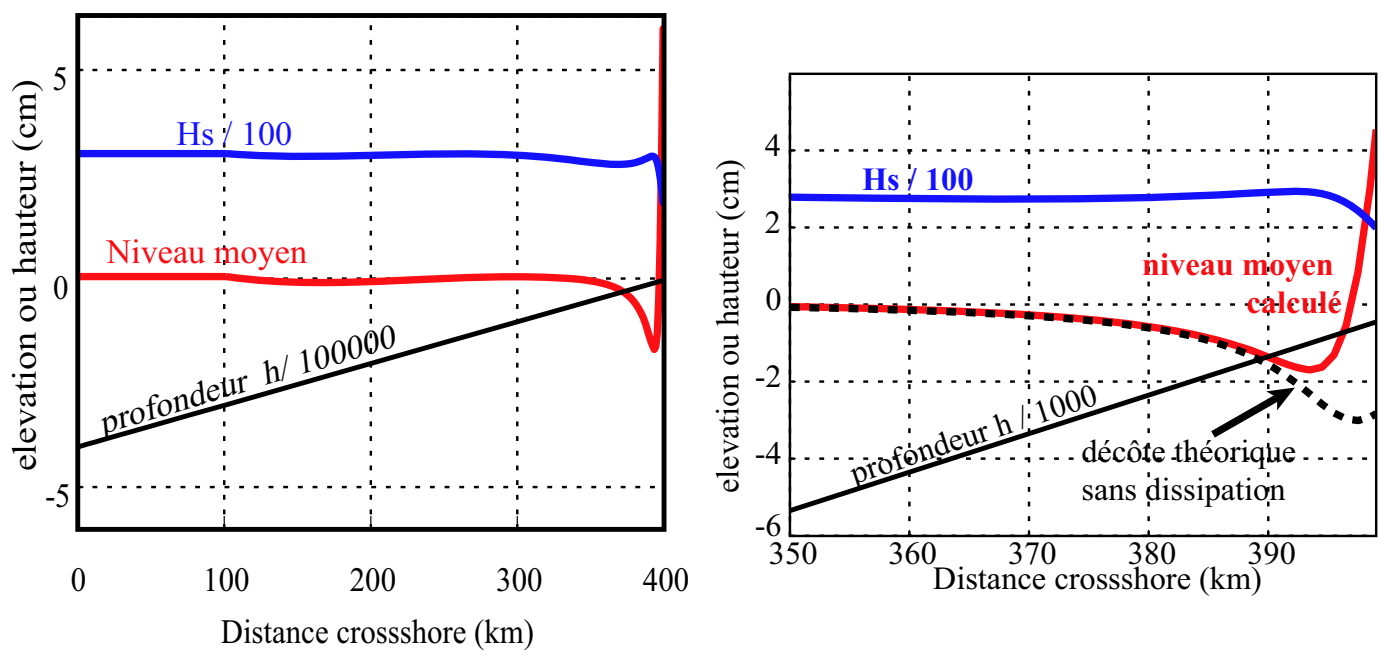

FIG. 2 - Variation de la surface libre sur le plateau continental (à gauche) et agrandissement de la zone littorale (à droite).

On retrouve la forme théorique de la décôte ${ }^{15}$, en dehors de la zone de déferlement.
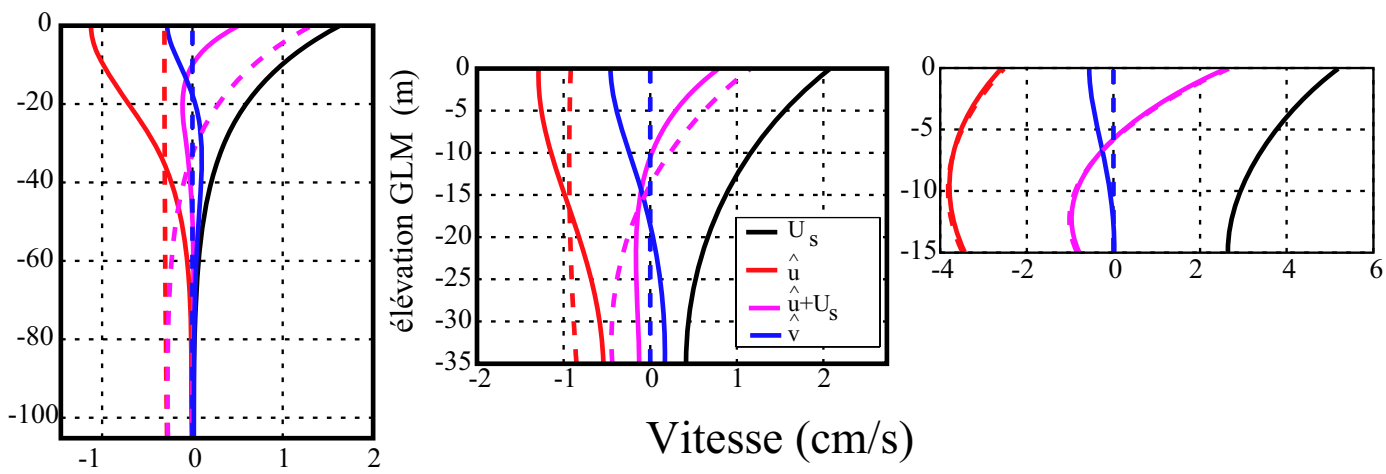

FIG. 3 - Profils de courant quasi-Eulérien (en rouge et bleu) et Lagrangien (en rose), par 110,35 et $15 \mathrm{~m}$ de fond.

Les calculs ont été réalisés en référentiel tournant (trait plein) et imobile ( $f_{3}=0$ pointillés) .

\section{Perspectives}

Le formalisme présenté ici est a priori applicable à la zone de déferlement et ses abords, et permet une description rationnelle des interactions vagues-courantturbulence. Elle évite le défaut principal des moyennes Eulérienne, qui est l'absence de définition physique du courant moyen entre crêtes et creux, au prix d'une petite complication mathématique. Toutefois, il est probable que l'approximation au second ordre en pente des vagues soit insuffisante en zone de déferlement. En particulier les vagues limites de Miche $^{18}$ donnent des cisaillements importants de la dérive de Stokes, même par faible profondeur. Un calcul des forçages GLM pour de telles vagues monochromatiques sur le point de déferler permettra d'évaluer l'er- 
reur réalisées dans le cadre de l'approximation linéaire. Pour des vagues aléatoires, il est possible d'étendre l'approximation à l'ordre supérieur, ce qui fera apparaitre les couplages de phases entre les différentes ondes, et, pour des vagues aléatoires, le bispectre. Ce dernier peut être calculé par des modèles spectraux fondés sur les équations de Boussinesq ${ }^{11}$ ou les équations d'Euler ${ }^{12}$.

\section{Bibliographie}

[1] Y. C. Agrawal, E. A. Terray, M. A. Donelan, P. A. Hwang, A. J. Williams, W. Drennan, K. Kahma, and S. Kitaigorodskii, "Enhanced dissipation of kinetic energy beneath breaking waves," Nature, vol. 359, pp. 219-220, 1992.

[2] D. G. Andrews and M. E. McIntyre, "An exact theory of nonlinear waves on a Lagrangian-mean flow," J. Fluid Mech., vol. 89, pp. 609-646, 1978.

[3] F. Ardhuin, Etat de la mer et dynamique de l'océan superficiel. Mémoire d'habilitation à diriger des recherches, Université de Bretagne Occidentale, Brest, France, 2005.

[4] F. Ardhuin and A. D. Jenkins, "On the interaction of surface waves and upper ocean turbulence,” J. Phys. Oceanogr., vol. 36, no. 3, pp. 551-557, 2006.

[5] J. Battjes and M. Stive, "Calibration and verification of a dissipation model for random breaking waves," J. Geophys. Res., vol. 90, no. C5, pp. 9159-9167, 1985.

[6] A. J. Bowen, "The generation of longshore currents on a plane beach," J. Mar. Res., vol. 27, pp. 206-215, 1969.

[7] C. Garrett, "Generation of Langmuir circulations by surface waves - a feedback mechanism," J. Mar. Res., vol. 34, pp. 117-130, 1976.

[8] J. Groeneweg, Wave-current interactions in a generalized Lagrangian mean formulation. PhD thesis, Delft University of Technology, The Netherlands, 1999.

[9] K. Hasselmann, "Wave-driven inertial oscillations," Geophys. Fluid Dyn., vol. 1, pp. 463-502, 1970.

[10] T. H. C. Herbers and M. C. Burton, "Nonlinear shoaling of directionally spread waves on a beach," J. Geophys. Res., vol. 102, no. C9, pp. 21,101-21,114, 1997.

[11] T. T. Janssen, T. H. C. Herbers, and J. A. Battjes, "Generalized evolution equation for nonlinear surface gravity waves over two-dimensional topography," J. Fluid Mech., vol. 552, pp. 393-418, 2006.

[12] K. E. Kenyon, "Stokes drift for random gravity waves," J. Geophys. Res., vol. 74, pp. 6991-6994, 1969.

[13] J. T. Kirby and T.-M. Chen, "Surface waves on vertically sheared flows : approximate dispersion relations," J. Geophys. Res., vol. 94, no. C1, pp. 1013-1027, 1989.

[14] S. Lentz, R. T. Guza, S. Elgar, F. Feddersen, and T. H. C. Herbers, "Momentum balances on the North Carolina inner shelf," J. Geophys. Res., vol. 104, no. C8, pp. 18205-18226, 1999.

[15] M. S. Longuet-Higgins and R. W. Stewart, "A note on wave set-up," J. Mar. Res., vol. 21, pp. 4-10, 1963.

[16] J. C. McWilliams, J. M. Restrepo, and E. M. Lane, "An asymptotic theory for the interaction of waves and currents in coastal waters," J. Fluid Mech., vol. 511, pp. 135178, 2004. 
[17] G. Mellor, "The three-dimensional current and surface wave equations," J. Phys. Oceanogr., vol. 33, pp. 1978-1989, 2003.

[18] A. Miche, "Mouvements ondulatoire de la mer en profondeur croissante ou décroissante. forme limite de la houle lors de son déferlement. application aux digues maritimes. troisième partie. forme et propriétés des houles limites lors du déferlement. croissance des vitesses vers la rive," Annales des Ponts et Chaussées, vol. Tome 114, pp. 369-406, 1944.

[19] N. Rascle, F. Ardhuin, and E. A. Terray, "Drift and mixing under the ocean surface. part $1:$ a coherent one-dimensional description with application to unstrati.ed conditions," J. Geophys. Res., vol. 111, p. C03016, 2006. doi :10.1029/2005JC003004.

[20] A. Shchepetkin and J. McWilliams, "The regional oceanic modeling system : A splitexplicit, free-surface, topography-following-coordinate ocean model," Ocean Modelling, vol. 9, no. C3, pp. 347-404, 2004. doi :10.1029/2001JC001047.

[21] J. A. Smith, "Wave-current interactions in finite-depth," J. Phys. Oceanogr., vol. 36, p. in press, 2006.

[22] E. B. Thornton and R. T. Guza, "Transformation of wave height distribution," J. Geophys. Res., vol. 88, no. C10, pp. 5,925-5,938, 1983.

[23] D. J. R. Walstra, J. Roelvink, and J. Groeneweg, "Calculation of wave-driven currents in a 3D mean flow model," in Proceedings of the 27th international conference on coastal engineering, Sydney, vol. 2, pp. 1050-1063, ASCE, 2001.

[24] Z. Xu and A. J. Bowen, "Wave- and wind-driven flow in water of finite depth," J. Phys. Oceanogr., vol. 24, pp. 1850-1866, 1994. 